\title{
Auto-reinforce Magnetic Flywheel as Recent Advancement of Automobile Flywheel
}

\author{
Ahmad Radikal Akbar ${ }^{\mathrm{a}}$ and Mokhtar Awang ${ }^{\mathrm{b}}$ \\ Dept. of Mechanical Engineering, Universiti Teknologi PETRONAS, Perak, Malaysia
}

\begin{abstract}
A new feature for flywheel energy storage device is proposed, considering the deficiencies on existing technology. This feature is introduced as auto-reinforce performance which means an ability to recover the kinetic energy after speed-down occurred as impact of sudden loading or sudden braking. The performance will significantly keep longer the stored energy of a flywheel device. This novel concept of flywheel is engineered by installing a number of Permanent Magnets (PM). The magnetism configuration such magnetic strength, magnetic energy density, pole direction, geometry, and dimension are influential parameters to its performance. By executing Finite Element Magnetic Modeling, it is possible to predict the design parameters such magnetic force and magnetic torque. Finally by evaluating these mechanical parameters, the key performance of this device such as percentage of energy reinforcement and percentage of discharge elongation can be predicted for prototyping references.
\end{abstract}

\section{Introduction}

Auto-reinforce Magnetic Flywheel (AMFLY) is constructed from two pieces of flywheel discs which is assembled in one shaft. Each flywheel has a special function and rotates independently to another. The first part is Primary Flywheel (PF) which acts as energy storage part, while the second part is Secondary Flywheel (SF) which serves as energy enhancer part. AMFLY works by employing some Permanent Magnets (PM) mounted in both PF and SF part. PM will interact attractively or repulsively (optional), which will give mechanical response to the system. When the load is applied to the shaft of flywheel, the load receives an amount of energy from PF which explains the cutback on PF's speed or stored energy. Immediately the SF will then raise-back the PF's kinetic energy by applying SF's magnetic coupling and SF's inertial mass. With this reinforce feature, presently this device is not only acts as energy storage, but also energy enhancer device. To prove this novel concept, a lab scale model has been developed as illustrated in Fig. 1a and Fig 1b.

\footnotetext{
${ }^{a}$ Corresponding author : radikal_g02063@utp.edu.my

b.mokhtar_awang@petronas.com.my
} 


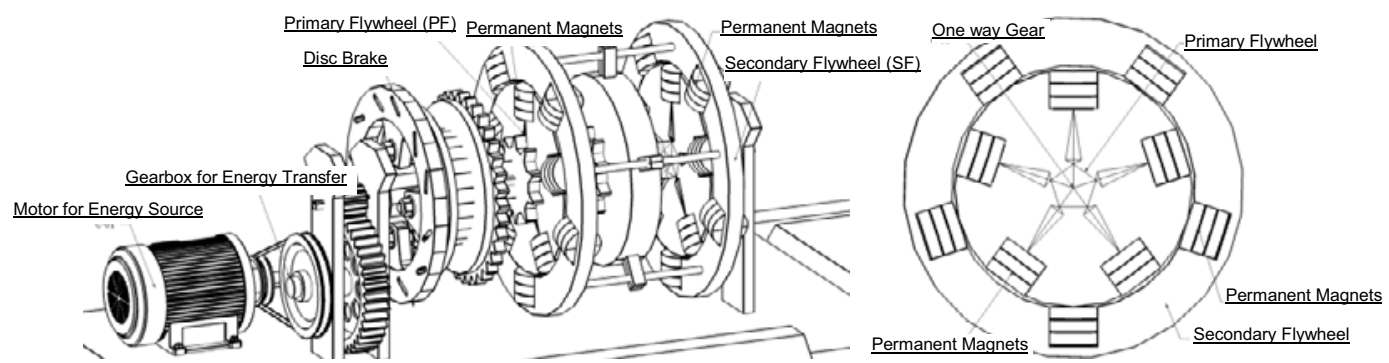

Figure 1. The AMFLY model: a. Overall 3D view; b. Dual magnetic flywheel discs

\section{Research Consideration}

\subsection{Mathematic of Magnetic Flywheel}

The kinetic energy stored in a flywheel ( $E_{k}$ in Joule) is proportional to the moment of inertia $(I)$ and the square of its rotational speed $(\omega)$, while the moment of inertia for any object is a function of its mass $(\mathrm{m})$ and its shape (radius and thickness) as Eq. 1 stated [1,2]. The important parameters of flywheel are its radius and its angular speed [3,4]. Eq. 1 shows that the most efficient way to increase the stored energy is to optimize the angular speed the flywheel $[5,6]$.

$$
E_{k}=\frac{1}{2} I \omega^{2}=\frac{1}{2}\left(\frac{1}{2} m r^{2}\right) \omega^{2}
$$

In case of magnetic part usage, some parameters should be considered such as magnetic flux $(B)$, magnetic force $(F)$, magnetic Torque $(T)$, and magnetic energy $\left(E_{m}\right)$. Magnetic force can be calculated by current-carrying conductor method, the total force is given by integrating the Lorentz equation at certain volume as Eq. 2 stated [7]. And the total torque about a point P is stated as Eq. 3 [8].

$$
\begin{gathered}
F=\int J \cdot B \cdot d V=\frac{\mu_{0} \cdot H^{2} \cdot A}{2}=\frac{B^{2} \cdot A}{2 \mu_{0}} \\
T=\int r \cdot J \cdot B \cdot d V
\end{gathered}
$$

Where, $A$ is the area of each surface (in $\mathrm{m}^{2}$ ); $H$ is their magnetizing field (in $\mathrm{A} / \mathrm{m}$ ); $\mu_{0}$ is the permeability of space $\left(4 \pi \cdot 10^{-7} \mathrm{~T} . \mathrm{m} / \mathrm{A}\right) ; B$ is the flux density (in Tesla); $r$ is the radius vector from $\mathrm{P}$ to the volume element, and the integral is taken over the volume of the conductor/magnetic material.

Some equations above are useful for analytical calculation but take long time to run on these problems [9]. Alternatively another applicable equation that widely has been used to perform faster calculation is co-energy method which is stated by Eq. 4 [9], where $w^{\prime}$ is co-energy while $w$ is energy.

$$
W^{\prime}=\int w^{\prime} \cdot d V=\int\left(B \cdot H-\int H \cdot d B\right) d V
$$

If two models are constructed which differ by $\Delta s$ in a distance $s$, then the force may be calculated from equation Eq. 4 above by taking the difference of the co-energy values as Eq. 5 stated. A similar equation holds for torque in terms of an angular displacement $\theta$ as stated by Eq. 6 [10].

$$
\begin{aligned}
& F_{s}=\frac{\partial W^{\prime}}{\partial s} \approx \frac{\Delta W^{\prime}}{\Delta s} \\
& T_{\theta}=\frac{\partial W^{\prime}}{\partial \theta} \approx \frac{\Delta W^{\prime}}{\Delta \theta}
\end{aligned}
$$

\subsection{Computational Analysis of Magnetic Flywheel}

To calculate magnetic global parameters on PM, several approaches among Finite Element Analyses (FEA) can be practiced. They are based on Maxwell's stress tensor, based on the virtual work, or based on the equivalent source models [11]. The method of virtual work is widely used to compute magnetic force distributions in permeable materials using Finite Element Magnetic Modeling (FEMM) [12]. For this particular consideration, the authors choose ANSYS Magnetostatic to carry out 3D analysis and MagNet Infolytica for 2D analysis. 


\subsection{Device Construction based on Predictive Study}

In this experimental stage, a lab scale model is engineered based on the following configurations in Table 1 which are possible to be setup. The following specified parameters are predicted through computational optimization.

Table 1. The specified properties of magnetic flywheel parameters

\begin{tabular}{cllclll} 
No & Magnetic Parameters & Value & No & Magnetic Parameters & Value \\
\hline 1 & Type / Strength of magnets & Neodymium & 6 & Radius of interaction & $20 \mathrm{~cm}$ \\
\hline 2 & Number of pairs & 5 pairs & 7 & Distance of magnets & $1 \mathrm{~cm}$ \\
\hline 3 & Pole / Interaction & Repulsive & 8 & Angle of magnetic vector & $0^{0}$ \\
\hline 4 & Shape of magnets & Bar & 9 & Periphery among magnets & $72^{0}$ \\
\hline 5 & Placement of magnets & Tangentially & 10 & Inertial mass of PF and SF & $1 \mathrm{~kg}$ and $3 \mathrm{~kg}$ \\
\hline
\end{tabular}

Furthermore, to emphasize the novelty of auto-reinforce feature, a comparative study also conducted to examine AMFLY vs. a conventional flywheel. The expected result is to show up the advantages of this novel device compare to the conventional one. The particular assessments will be energy capacity, energy transfer, charge time, and discharge time. An extra outcome in these criterions will lead AMFLY among other flywheel technologies.

\section{Performance Prediction}

\subsection{Prediction of Magnetic Performance}

Finite Element Method Magnetics has contributed in visualizing the stored energy E, flux density B, vector flux $\varphi$, and flux of line $\varphi$ respectively which are gathered in Fig. 2 . In this figure, the $100 \%$ amount of energy density on the leftmost column constitutes to the quantity of the maximum torque of SF to PF. When SF shifts relatively to PF (state II-III-IV), its maximum torque is dropping. This is because at these positions, the energy density surrounding the device is decreasing due to the negative flux vector accumulation since its interaction is repulsive. Nevertheless, the initial potential magnetic energy would not reduce though PM performed mechanical interaction all the time [13].

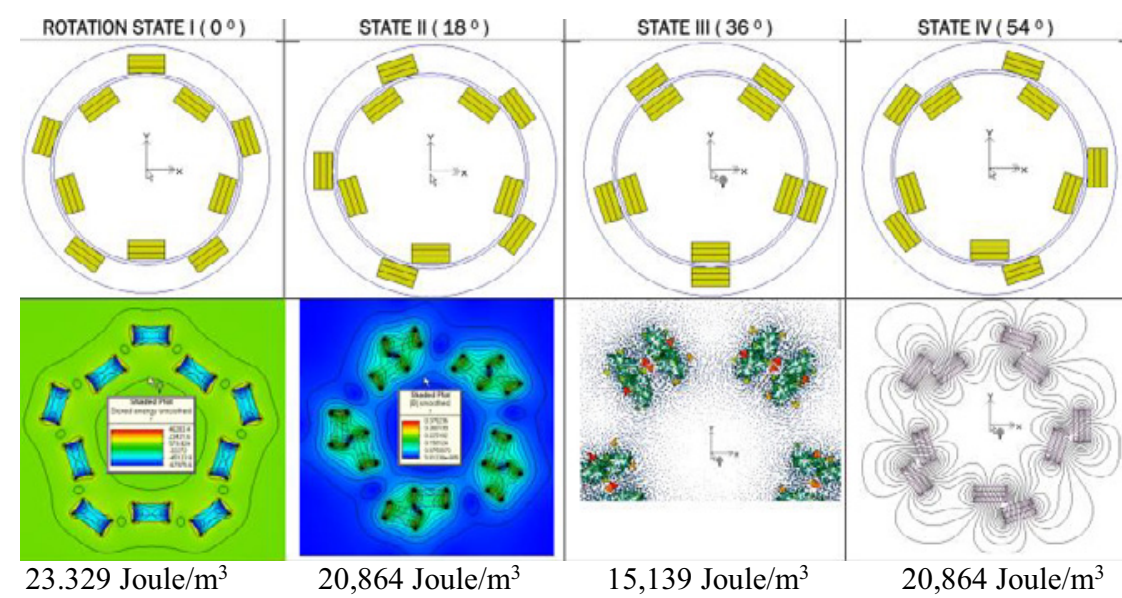

Figure 2. 2D simulation by MagNet Infolytica in 4 states of rotation and 4 kinds of visualization: a. contour of stored energy (E); b. contour of flux density (B); c. flux vector ( $\varphi$ ); d: flux line $(\varphi)$

Those interpretations were explained more by another magnetism simulator, ANSYS Magnetostatic in $3 \mathrm{D}$ boundary as shown in Fig. 3. Visualization of the contour and vector are describing the percentage of PM body needed to drive the torque of SF to PF. 

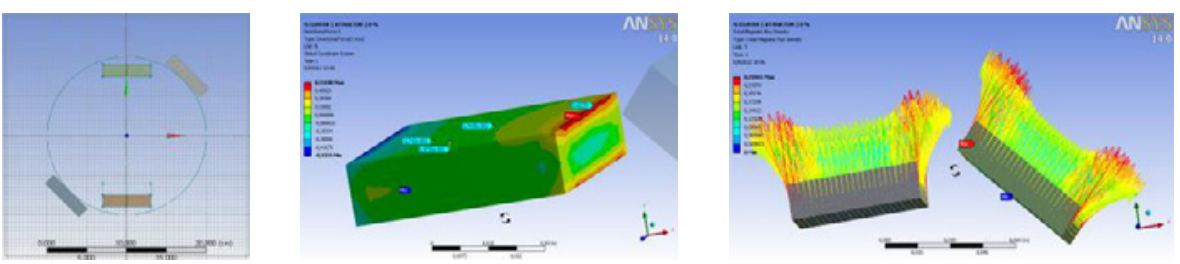

Figure 3. 3D simulation by ANSYS: a. rotating geometries; b. contour and probe of $H$; c. vector of flux $\varphi$

\subsection{Prediction of Mechanical Performance}

It is also possible to predict mechanical properties using a magnetism simulator. MagNet Infolytica and ANSYS Magnetostatic are having a feature to compute few mechanical equations such magnetic force $(\mathrm{F})$ and magnetic torque $(\mathrm{T})$ that present in the magnetic circuits. PM configuration introduced in Fig. 2 has been simplified into 1 pair ( 2 permanent magnets) interaction due to the software operation, as adapted in Fig. 4 below.
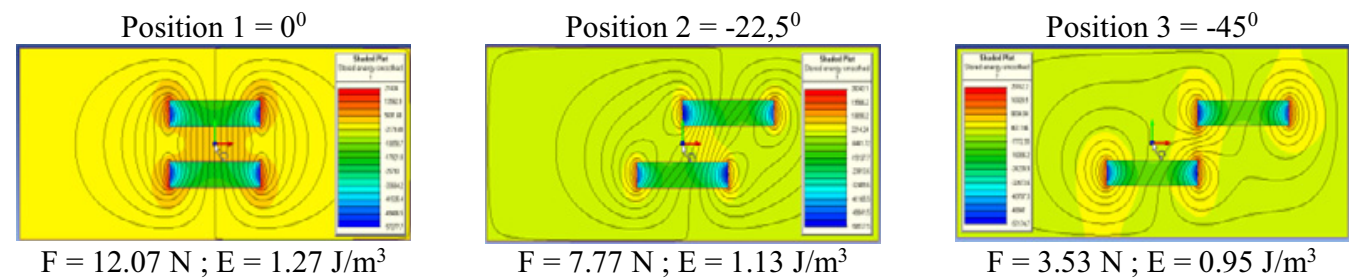

Figure 4. Translational motion which is adapted from rotational motion

In this attraction mode, both of magnets are producing forces which are accumulated as resultant force. The strongest interaction is at position $0^{0}$ which means SF may attract PF with maximum magnetic force. Additionally, ANSYS magnetostatic is presenting more detail of force accumulation as shown in Fig. 5. This accumulative force can be directly used to calculate the torque of SF to PF. This operation will be practiced to predict the minimum torque of SF needed to drive inertial mass of PF.
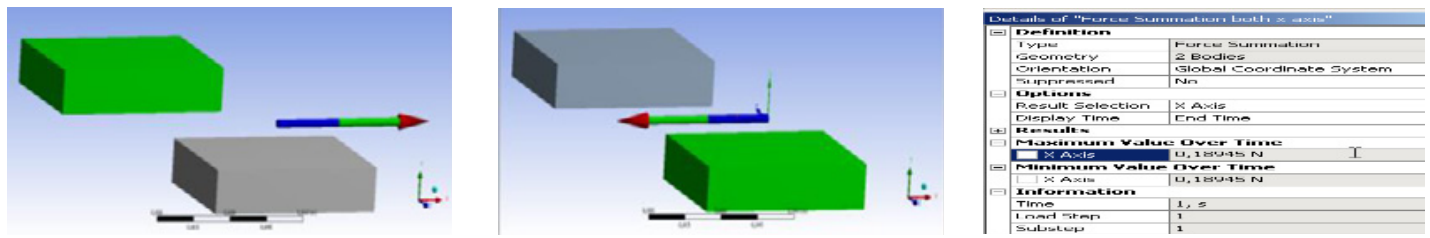

Figure 5. Resultant of force by: a. top magnet; b. bottom magnet; c. both magnets.

\subsection{Prediction of Auto-reinforce Feature}

The optimum criterion of this device will be: 1) percentage of reinforcement; and 2) percentage of discharge elongation. Fig. 6 recorded the speed over time of PF and SF in 200 seconds. The speed of $\mathrm{PF}$ is represented by the diamond dotted line while the cubical dotted line represents SF speed. At the beginning during charging phase, the PF's speed and SF's speed are increasing by slight delay, around 10-15 seconds. This is the time taken for the magnetic interaction (magnetic repulsion) to react, means the PM induced the SF to rotate until SF reach the same speed of PF. Focusing $50^{\text {th }}$ to $70^{\text {th }}$ seconds, after the PF declines in speed, there is a slight rise. At the same time, the SF is at peak point (maximum speed). At this point, the load is applied, which explains a reduction in speed or deceleration is occurring during this discharging phase. Shortly, the SF's speed surpasses the speed of PF. Subsequently at this point, the inertial mass of SF and its magnetic coupling will drive a motion to PF till their speeds are coincident. In the graph, a rise curve of PF indicates the presence of reinforcement. During this reinforcement action, the percentage of raising energy is about $12 \%$ of initial decrement magnitude. On the other hand, the right-hand graph shows the normal trend of 
conventional flywheel. This common one has a simple performance curve since it is constructed by only one part (primary disc) connected to either motor or load.
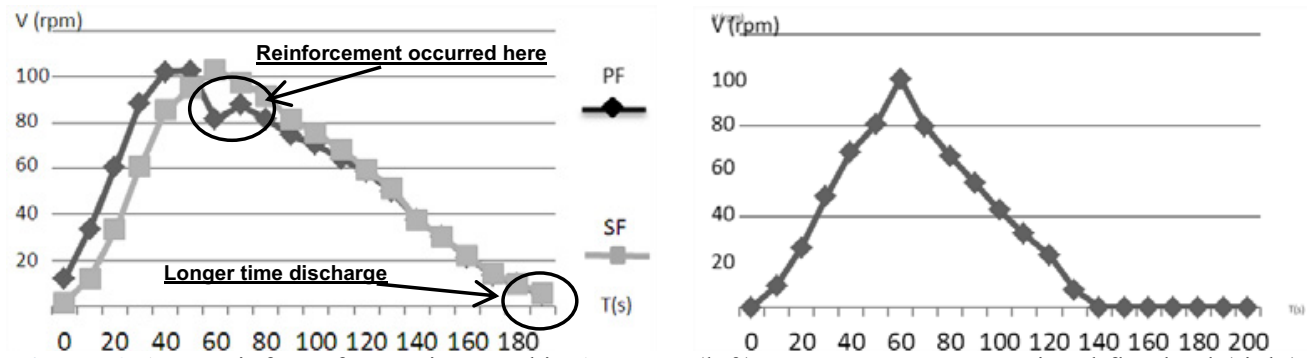

Figure 6. Auto-reinforce feature invented in AMFLY (left), compare to a conventional flywheel (right);

Since the area under the graph indicates a capacity of energy stored in flywheel, it can be deduced that AMFLY device has higher energy capacity compare to the common flywheel technology. Finally, based on this prediction study, authors have developed a lab scale prototype as shown in Fig. 7.
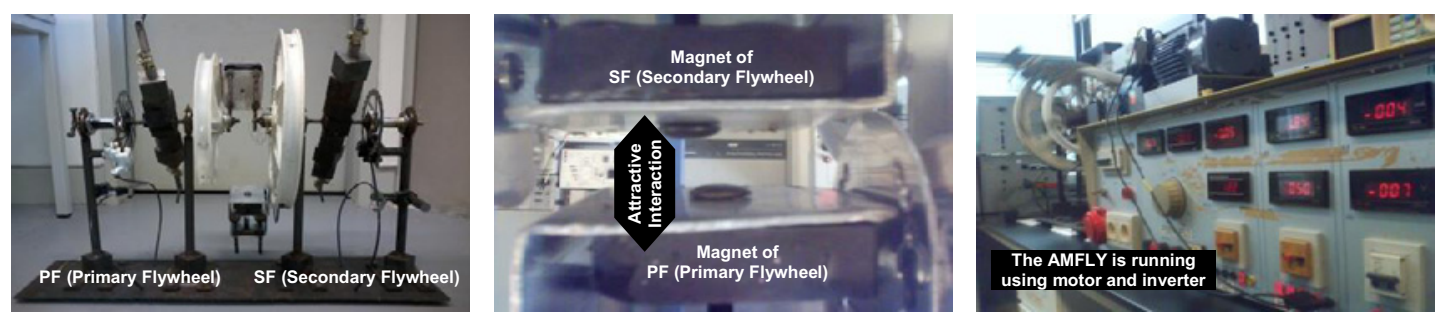

Figure 7. Lab-scale prototype: a. Overall (PF and SF parts); b. PM interaction; c. Running test.

\section{Conclusion}

Research on magnetic properties has brought practical clues, i.e.:

1) Geometry of PM should be wider (not thicker) in order to provide a longer interaction rather than a stronger interaction. 2) Periphery of magnetized part should be prolonged in order to provide longer time of interaction since the device is rotating in high velocity.

In line with that, research on mechanical properties has guided into technical suggestion, i.e.:

1) Inertial moment of both of flywheels (PF and SF) must be properly specified with the available torque by PM. 2) The energy reinforcement and discharge elongation can be deduced after the torque of SF to PF is already predicted.

\section{Acknowledgment}

The authors are grateful for the Fundamental Research Grant Scheme/FRGS from MOHE Malaysia (2013-0153AB-106).

\section{References}

[1] R. Peña-Alzola., International Conference on Power Engineering, Energy and Electrical Drives. Spain. May 2011

[2] Christopher A. Haller., Dynamic Energy Management using Flywheel Energy Storage Systems: Design \& Applications. ECE 530 Contemporary Energy Applications (Fall 2008)

[3] Bjo"rn Bolund, Hans Bernhoff, Mats Leijon., Renewable and Sustainable Energy Reviews 11, 235-258 (2007)

[4] H. Ibrahim, A. Ilinca, J. Perron., Renewable and Sustainable Energy Reviews 12, 1221-1250 (2008)

[5] Arslan, Mehmet Ali, Materials \& Design 29 (2), 514-518 (2008)

[6] Alan Ruddell, Investigation on Storage Technologies for Intermittent Renewable Energies: Evaluation and recommended R\&D strategy. Storage Technology Report. (2003) 
[7] R. Sánchez GrandíaI, IEEE Transactions on Magnetics, 44 (9) (2008)

[8] Santiago Sanz, IEEE Transactions on Applied Superconductivity, 20 (3), 2010

[9] GY Jeong, IEEE Transactions on Magnetics, 47 (10) (2011)

[10] Olivier Barré, IEEE Transactions on Magnetics, 42 (4) (2006)

[11] W. N. Fu.. IEEE Transactions on Magnetics, 40 ( 2) (2004)

[12] Francois Henrotte, Journal of Comp and Applied Math 168, 235-243 (2004)

[13] Ahmad Radikal Akbar, Mokhtar Awang, ICAMN III. Scientific Net, (2013) 